\title{
Cytokine responses to Staphylococcus aureus bloodstream infection differ between patient cohorts that have different clinical courses of infection
}

\author{
Sinead McNicholas', Alida Fe Talento², Joanne O'Gorman, Margaret M Hannan³, Maureen Lynch³, \\ Catherine M Greene ${ }^{4}$, Hilary Humphreys ${ }^{1,2}$ and Deirdre Fitzgerald-Hughes ${ }^{1 *}$
}

\begin{abstract}
Background: The clinical course of Staphylococcus aureus bloodstream infection is unpredictable and bacterial virulence, host immune response and patient characteristics are among the factors that contribute to the clinical course of infection. To investigate the relationship between cytokine response and clinical outcome, circulating cytokine levels were investigated in response to S. aureus bloodstream infection in patients with different clinical courses of infection.

Methods: A prospective study was carried out in 61 patients with S. aureus bloodstream infection and circulating levels of IL-6, GRO- $\gamma$, RANTES and leptin were assessed over the course of the infection. Levels were compared in patients with complicated courses of infection (e.g. infective endocarditis) versus uncomplicated courses of $S$. aureus bloodstream infection and methicillin-resistant $S$. aureus Vs methicillin-susceptible $S$. aureus infection.

Results: Significantly lower leptin levels $(p<0.05)$ and significantly higher IL-6 levels $(p<0.05)$ were detected at laboratory diagnosis in patients with complicated compared to uncomplicated S. aureus bloodstream infection. Significantly higher levels of GRO- $y$ were associated with MRSA infection compared to MSSA infection.
\end{abstract}

Conclusions: IL-6 may be an early inflammatory marker of complicated S. aureus bloodstream infection. Leptin may be protective against the development of a complicated $S$. aureus bloodstream infection.

Keywords: Staphylococcus aureus, Bloodstream infection, Pro inflammatory cytokine, Renal impairment, Haemodialysis, Immune mediator, Leptin, RANTES, IL-6

\section{Background}

Staphylococcus aureus bloodstream infection (SABSI) is one of the most severe manifestations of $S$. aureus with an estimated mortality rate of $20 \%$ [1]. However, the outcome of SABSI is variable. Many patients develop uncomplicated resolving infections that respond to appropriate antimicrobial therapy while others develop persistent or complicated infection with metastatic foci (e.g. infective endocarditis (IE)), which may be fatal. Factors that contribute to the clinical outcome of SABSI, include the

\footnotetext{
* Correspondence: dfitzgeraldhughes@rcsi.ie

'Department of Clinical Microbiology, RCSI Education and Research Centre, Beaumont Hospital, Dublin 9, Ireland

Full list of author information is available at the end of the article
}

virulence and antibiotic susceptibility of the infecting isolate $[2,3]$, host innate and humoral immune responses $[4,5]$ and the underlying condition of the patient [6]. S. aureus molecules such as peptidoglycan and lipoteichoic acid (LTA) are potential stimulators of cytokine production (e.g. TNF- $\alpha$, IL-1 $\beta$, IL-6, IL-4, IL-8, IFN- $\gamma$ and IL-12), in response to infection [7] but un-regulated cytokine production may contribute to $S$. aureus pathogenesis. Differential cytokine responses to $S$. aureus may contribute to varying clinical outcomes of SABSI. This prospective study aimed to; identify cytokines or chemokines that are important in the immune response to SABSI, determine the levels of these molecules in sequential plasma samples taken over the course of SABSI and investigate these

\section{Ciomed Central}

(c) 2014 McNicholas et al.; licensee BioMed Central Ltd. This is an Open Access article distributed under the terms of the Creative Commons Attribution License (http://creativecommons.org/licenses/by/2.0), which permits unrestricted use, distribution, and reproduction in any medium, provided the original work is properly credited. The Creative Commons Public Domain Dedication waiver (http://creativecommons.org/publicdomain/zero/1.0/) applies to the data made available in this article, unless otherwise stated. 
cytokine responses in SABSI with a complicated clinical course versus an uncomplicated course and those with MRSA infection compared to those with MSSA infection.

\section{Methods}

\section{Setting, patients and definitions}

Blood samples were collected from patients with SABSI in Beaumont Hospital (BH), from October 2008 to February 2011 and in the Mater Misericordiae University Hospital (MMUH) from November 2009 to March 2010. Both are $>500$-bed tertiary referral centres, located in Dublin, Ireland. $\mathrm{BH}$ is the national centre for renal and pancreatic transplantation, neurosurgery and cochlear implantation. The MMUH has the largest cardiothoracic surgical department in the country and is the national centre for heart and lung transplantation. Approval from $\mathrm{BH}$ and MMUH Ethics Committees was received. Participation was by informed consent/assent from patients/relatives. Patient demographics, including age, sex, co-morbidities, timing of onset of SABSI, source of SABSI, isolate antimicrobial susceptibility and infection outcomes were collected prospectively from patient's charts, nursing notes and microbiological records. Plasma samples were prepared from blood collected on the day of the laboratory diagnosis of SABSI, seven days later and in patients with complicated infection, on day 14 after laboratory-confirmed diagnosis. Plasma was also prepared from the blood of four healthy control individuals. An uncomplicated course of infection was defined specifically for this study as follows; negative follow-up blood culture, subsidence of fever within $72 \mathrm{~h}$, normal findings on transesophageal echocardiogram and no symptoms suggestive of metastatic infection. A complicated course of infection was defined specifically for this study as; positive follow up blood culture, despite at least three days of appropriate antibiotics (e.g. flucloxacillin for methicillin-susceptible $S$. aureus (MSSA), vancomycin for methicillin-resistant S. aureus (MRSA)), disseminated infection such as osteomyelitis, or IE. Nosocomial, healthcareassociated and community-acquired infection were defined as described by Friedman et al. [8].

\section{Preliminary identification of cytokines in plasma from patients with SABSI using a panel of pro-inflammatory cytokines}

A commercially available antibody based cytokine/ chemokine microarray (RayBio ${ }^{\circledR}$ Human Cytokine Antibody Array 3 (RayBiotech Inc., GA, USA)) was used to identify cytokines that were differentially expressed in pooled plasma (taken on the day of diagnosis) from three patients with uncomplicated BSI and three with complicated BSI. Pooled plasma was used for the preliminary screen only, to minimise resources required. The panel consists of antibodies against 42 pro-inflammatory cytokines and chemokines and the manufacturer's instructions were followed. Cytokine signal intensities were captured and quantified by the chemiluminescence imaging system G:BOX Chemi XL (Syngene UK, Cambridge, UK). Mean values for each cytokine were expressed relative to the mean positive value for each array membrane (mean of 6 positive control spots per membrane). A change in cytokine levels in pooled plasma, at a ratio threshold of at least 1.4 fold between groups was considered to be potentially relevant and the results from this preliminary screen influenced the decision on which cytokines to investigate in individual patients over the course of their infection.

\section{Enzyme-linked immunosorbant assay (ELISA)}

The levels of IL-6, GRO- $\gamma$, RANTES and leptin were determined in patient and healthy donor plasma samples using immunometric sandwich ELISA ( $R$ and D systems, Abington, UK (IL-6, RANTES, leptin) or Acris Antibodies Ltd, Germany (GRO- $\gamma$ ). The manufacturer's instructions were followed using 50-100 $\mu \mathrm{l}$ of plasma. Plasma protein concentrations were measured using the Bradford assay and cytokine levels were normalised to plasma protein concentration to account for variability in blood processing and biological variations in plasma protein concentrations between patients, many of whom were undergoing haemodialysis.

\section{Statistical analysis}

Unpaired $t$-tests were used to compare the means of data sets. Fisher's exact test was used to compare categorical data. Graphpad Prism 4 was used for Statistical analyses.

\section{Results}

\section{Patient details}

In total, 61 patients were studied, 15 (24.5\%) with MRSA BSI, 46 (75.4\%) with MSSA BSI. Fifty (82\%) patients had an uncomplicated BSI and 11 (18\%) had a complicated course of SABSI as defined for this study. Detailed patient demographics are shown in Table 1. Two patients died and SABSI was identified as the cause in one of these (complicated, IE). A high rate of IE was found among those with complicated SABSI (8/11, 73\%) and most complicated SABSI was healthcare-associated (7/ $11,64 \%)$. Of the 15 patients with MRSA infection, four had a complicated clinical course and an increased risk of complications was not identified in those with MRSA Vs MSSA (4/15, 26\% Vs, 11/46 (24\%), RR1.1).

\section{Identification of cytokines for further investigation in complicated or uncomplicated SABSI}

A panel of 42 cytokine antibodies were probed by hybridization of pooled plasma taken on the day of diagnosis, from three patients with complicated SABSI and three 
Table 1 Characteristics of 61 patients with S. aureus bloodstream infection (BSI)

\begin{tabular}{|c|c|}
\hline Patient characteristic & No. of patient (\%) \\
\hline \multicolumn{2}{|l|}{ Gender } \\
\hline Male & $38(62.3)$ \\
\hline Female & $23(37.7)$ \\
\hline \multicolumn{2}{|l|}{ Age } \\
\hline$>65$ & $35(57.38)$ \\
\hline$<65$ & $26(42.63)$ \\
\hline \multicolumn{2}{|l|}{ Co-morbidities $^{a}$} \\
\hline Cardiac disease & $22(36.07)$ \\
\hline${ }^{b}$ Renal impairment & $23(37.70)$ \\
\hline Diabetes mellitus & $16(26.23)$ \\
\hline Chronic obstructive pulmonary disease & $8(13.11)$ \\
\hline Autoimmune disease & $6(9.84)$ \\
\hline \multicolumn{2}{|l|}{ Onset of $\mathrm{BSI}^{\mathrm{C}}$} \\
\hline Nosocomial & $31(50.82)$ \\
\hline Healthcare-associated & $22(36.07)$ \\
\hline Community-acquired & $8(13.11)$ \\
\hline \multicolumn{2}{|l|}{ Source of BSI } \\
\hline Unknown & $15(24.6)$ \\
\hline Prosthetic device & $30(49.18)$ \\
\hline Other & $16(26.23)$ \\
\hline \multicolumn{2}{|l|}{ Susceptibility of infecting isolate } \\
\hline Methicillin-susceptible S. aureus & $46(75.41)$ \\
\hline Methicillin-resistant S. aureus & $15(24.59)$ \\
\hline \multicolumn{2}{|l|}{ Outcome of BSI } \\
\hline Uncomplicated BSI & $50(81.96)$ \\
\hline Complicated BSI & $11(18.03)$ \\
\hline Death & $2(3.28)$ \\
\hline \multicolumn{2}{|l|}{ Type of complication } \\
\hline Infective endocarditis & $8(13.11)$ \\
\hline Persistently positive blood culture & $1(1.64)$ \\
\hline Septic arthritis & $1(1.64)$ \\
\hline Discitis & $1(1.64)$ \\
\hline
\end{tabular}

${ }^{a}$ More than one co-morbidity was indentified in some patients. ${ }^{b}$ Of 23 patients with renal impairment, 21 were on haemodialysis (HD), one had chronic renal failure but was not on $\mathrm{HD}$ and one was receiving plasmaphoresis for Waldenströms macroglobulinaemia. ' ${ }^{\circ}$ Onset of BSI defined as described by Friedman et al., 2002 [8].

with uncomplicated SABSI. The position and name of each of the 42 cytokine antibodies on the array is shown on the cytokine antibody array map (Figure 1A). An image of the resulting hybridisation patterns for representative pooled samples from each group is shown in Figure 1B and cytokines that showed $\geq 1.4$ fold differences in levels between groups are highlighted. These cytokines; IL-6, GRO- $\gamma$ and RANTES (Regulated upon Activation, Normal T-cell Expressed, and Secreted) were identified for further study. Leptin levels were marginally lower in pooled plasma from patients with complicated SABSI compared to uncomplicated but this cytokine was further studied because its levels were increased $>2$-fold in pooled plasma from three patients with MRSA compared to MSSA BSI (Figure 1C).

Further investigation of these four cytokines in all patients with SABSI, using individual ELISAs revealed inter-patient variability in cytokine levels. However, the mean cytokine levels varied over the course of SABSI (Figure 2). Mean IL-6 and GRO- $\gamma$ levels decreased over the course of infection, and the decrease was statistically significant in the case of IL-6 $(p \leq 0.05)$. Mean RANTES levels were increased seven days following laboratory diagnosis compared to levels on the day of laboratory diagnosis of SABSI $(p \leq 0.0001)$. Further samples were taken 14 days following SABSI diagnosis only from patients with complicated SABSI $(\mathrm{n}=11)$ and were therefore not included in these analyses.

\section{Cytokine levels and the clinical outcome of SABSI}

The mean cytokine levels found in patients with complicated SABSI Vs uncomplicated SABSI are shown in Figure 3. Those with complicated SABSI had significantly higher mean IL-6 levels compared to those with uncomplicated SABSI on the day of diagnosis $(p \leq 0.05)$ (Figure 3, panel A). Mean GRO- $\gamma$ levels were greater in patients with complicated SABSI compared to uncomplicated SABSI but these differences were not statistically significant (Figure 3, panel B). Mean RANTES (Figure 3, panel C) levels were significantly higher on day seven following diagnosis of SABSI compared to those found on the day of diagnosis in patients with uncomplicated $(p \leq 0.0001)$ and complicated SABSI $(p \leq 0.05)$. Leptin levels (Figure 3, panel D) were significantly reduced in patients with complicated SABSI compared to patients with uncomplicated SABSI on the day of diagnosis $(p \leq 0.05)$. Mean levels of IL-6, GRO- $\gamma$ and RANTES values in healthy individuals were lower than those in patients with complicated and uncomplicated SABSI on the day of diagnosis and seven days later. Mean leptin levels, although lower in healthy individuals than those with uncomplicated SABSI, were raised in healthy individuals compared to those with complicated infection (Figure 3).

\section{Cytokine responses SABSI caused by MSSA Vs MRSA}

The mean levels of GRO- $\gamma$ were significantly greater in patient with SABSI in cases where an MRSA was the source of infection $(\mathrm{p} \leq 0.05)$ but this increase was only evident when comparing the samples taken on the day of diagnosis (Figure 4). The mean level of the other three cytokines investigated, were not significantly altered in patients with SABSI caused by MRSA Vs MSSA (data not shown). 


\begin{tabular}{|c|c|c|c|c|c|c|c|c|c|c|c|c|}
\hline & $A$ & B & $\mathrm{C}$ & D & $E$ & $F$ & G & $\mathrm{H}$ & $I$ & $\mathrm{~J}$ & K & $\mathbf{L}$ \\
\hline 1 & Pos & Pos & $\mathrm{Neg}$ & $\mathrm{Neg}$ & ENA-78 & GCSF & GM-CSF & GRO & GRO- $\alpha$ & $1-309$ & IL-1 $\alpha$ & IL-1 $\beta$ \\
\hline 2 & Pos & Pos & $\mathrm{Neg}$ & $\mathrm{Neg}$ & ENA-78 & GCSF & GM-CSF & GRO & GRO- $\alpha$ & $1-309$ & IL-1 $\alpha$ & IL-1 $1 \beta$ \\
\hline 3 & IL-2 & IL-3 & IL-4 & IL-5 & IL-6 & IL-7 & IL-8 & IL-10 & IL-12p40p70 & IL-13 & IL-15 & IFN- $\gamma$ \\
\hline 4 & IL-2 & IL-3 & IL-4 & IL-5 & IL-6 & IL-7 & IL-8 & IL-10 & IL-12p40p70 & IL-13 & IL-15 & $\mathrm{IFN}-\gamma$ \\
\hline 5 & MCP-1 & MCP-2 & MCP-3 & MCSF & MDC & MIG & MIP-1 $\delta$ & RANTES & SCF & SDF-1 & TARC & TGF- $\beta$ \\
\hline 6 & MCP-1 & MCP-2 & MCP-3 & MCSF & MDC & MIG & MIP-1 $\delta$ & RANTES & SCF & SDF-1 & TARC & TGF-b \\
\hline 7 & TNF- $\alpha$ & TNF- $\beta$ & EGF & IGF-1 & Angiogenin & Oncostatin M & Thrombopoietin & VEGF & PDGF BB & Leptin & $\mathrm{Neg}$ & Pos \\
\hline 8 & TNF- $\alpha$ & TNF- $\beta$ & EGF & IGF-1 & Angiogenin & Oncostatin M & Thrombopoietin & VEGF & PDGF BB & Leptin & $\mathrm{Neg}$ & Pos \\
\hline
\end{tabular}

B Uncomplicated SABSI Complicated SABSI

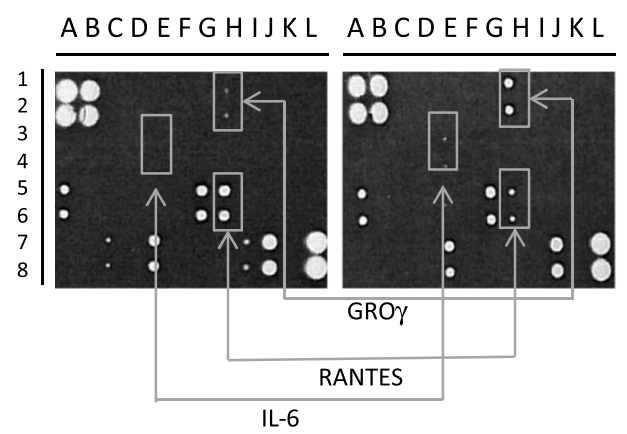

C MSSABSI

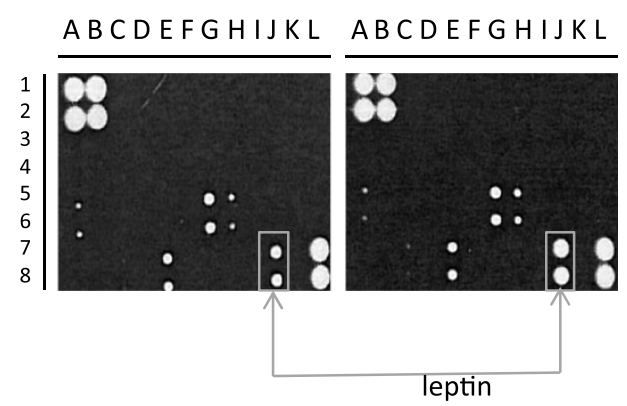

Figure 1 Analysis of differential cytokine levels in pooled plasma from patients with SABSI. The cytokine antibody array map (RayBiotech Inc., GA) showing the positions of 42 duplicate cytokines, positive and negative controls (A). Hybridization patterns for pooled plasma from three patients per group, comparing patients with complicated SABSI to patients with uncomplicated SABSI (B) and patients with MSSA to patients with MRSA (C). Arrows indicate cytokines selected for investigation in all patients (B and $\mathbf{C}$ ) and were based on a fold change in levels of $\geq 1.4$ between groups. Fold change was estimated from cytokine signal intensity from compared groups (uncomplicated/complicated, MSSA/MRSA) where signal intensity was normalised with respect to the signal intensity of six positive control spots on each membrane, located at positions $A 1, A 2, B 1, B 2, L 7, L 8$.

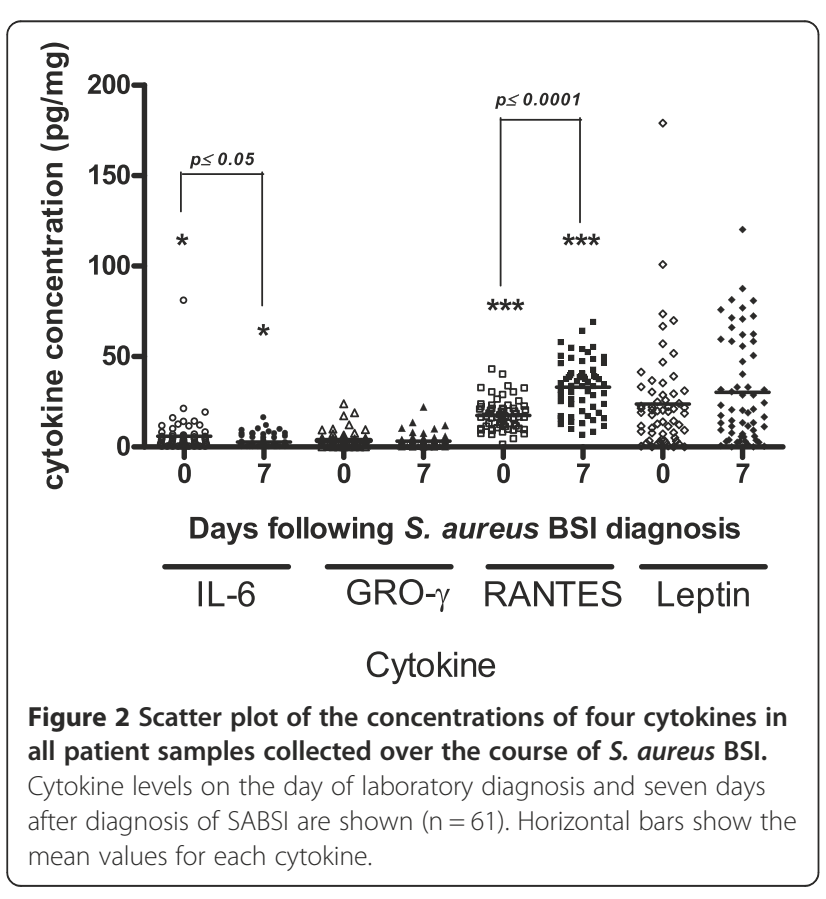

\section{Discussion}

Pro-inflammatory cytokines released in response to $S$. aureus infection may contribute to the cascade of events that lead to severe complications in SABSI [9]. Cytokines including IL-6, GRO- $\gamma$ and RANTES are up-regulated in response to $S$. aureus clinical isolates in epithelial cell infection models $[10,11]$. We observed changes in the circulating levels of these cytokines in patients over the course of SABSI. In addition, greater circulating IL-6 levels and reduced leptin levels were observed in complicated SABSI compared to uncomplicated SABSI. Complicated infection was defined specifically for this study as described above and IE was the predominant complication. The surprisingly high rate of IE $(8 / 61,13 \%)$ may be partly explained by our policy of echocardiograms for all patients with SABSI regardless of the response to therapy and the presence of a national centre for cardiac and lung transplantation in one of the study hospitals. In addition, an increased circulating level of GRO- $\gamma$ was noted in response to MRSA Vs MSSA bloodstream infection.

The cytokine response to sepsis has been well documented, with IL-6 suggested as an early marker and a marker of severity [12-15]. The cytokine response in 


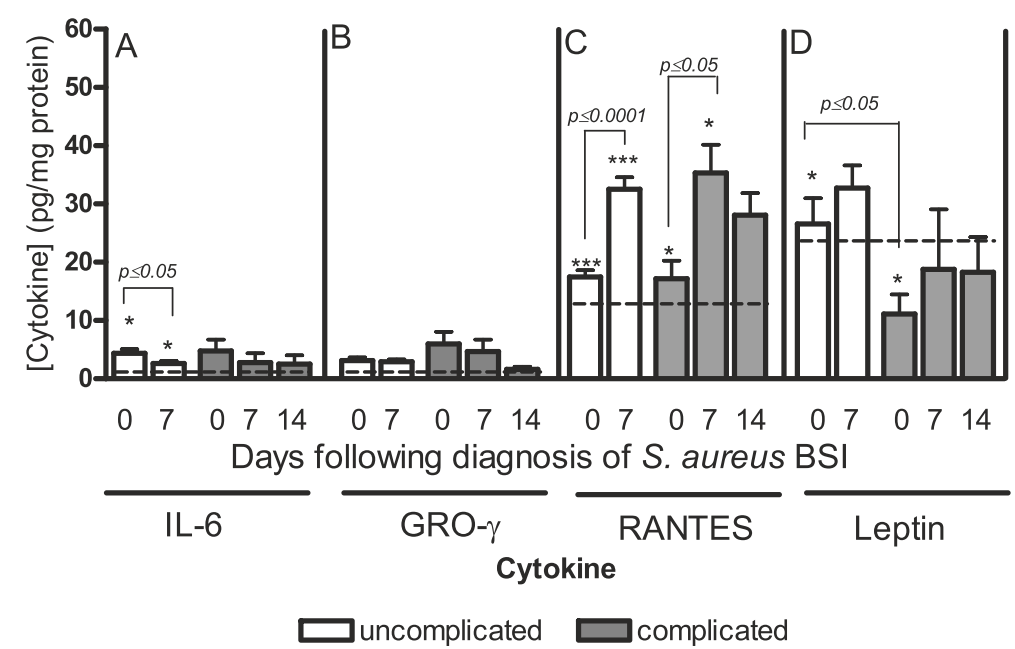

Figure 3 Cytokine levels in patients with uncomplicated Vs complicated SABSI. Cytokine levels in plasma from patients with uncomplicated (clear bars, $n=50$ ) and complicated (solid grey bars, $n=11$ ) SABSI at diagnosis (day 0), day 7 and day 14. Data shown represent mean cytokine concentration \pm SEM for IL-6 (A), GRO- $>$ (B), RANTES (C) and leptin (D). Dotted lines in each panel indicate the mean cytokine levels found in four healthy control individuals.

SABSI also appears to involve IL-6 elevation early in the course of infection although the levels were lower than those reported in Gram-positive sepsis [16]. An earlier study suggested that IL-6 levels decay more rapidly in sepsis survivors than in non-survivors over a $96 \mathrm{~h}$ period supporting its prognostic value [17]. However, in the present study on SABSI, where cytokines were assessed over a longer period, IL-6 levels decreased to similar levels in complicated and uncomplicated SABSI by day

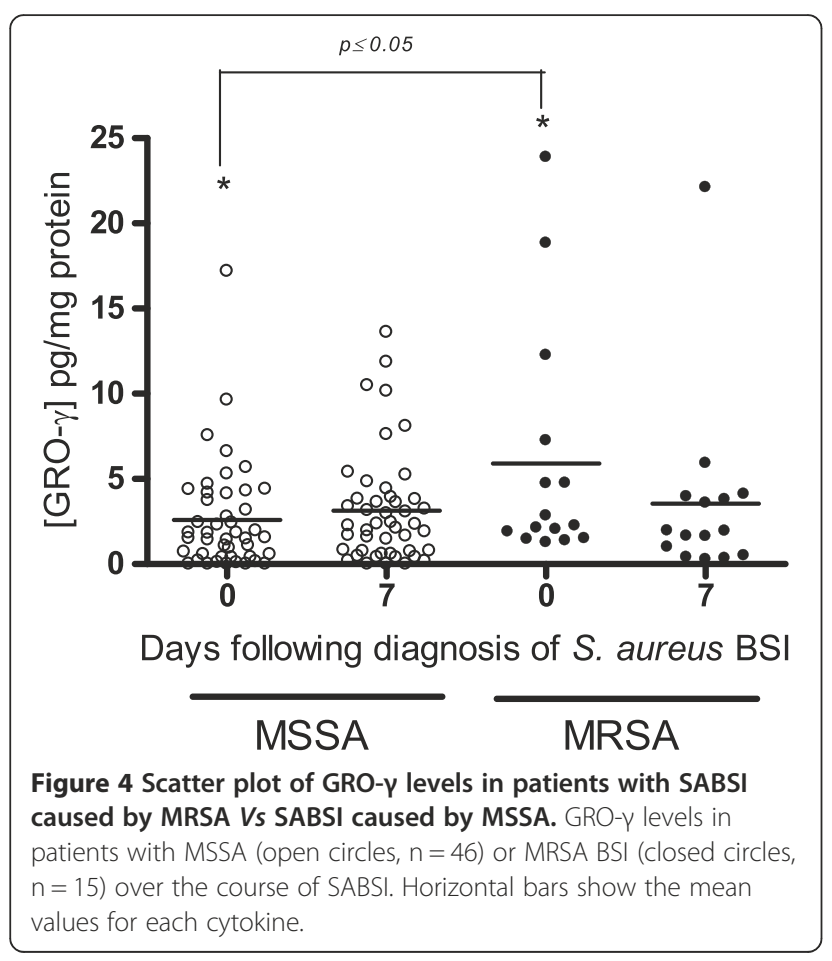

seven following laboratory diagnosis. Nonetheless, a significantly elevated IL-6 level on the day of diagnosis of SABSI appears to correlate with a poorer prognosis.

In-vitro studies have shown increased RANTES levels in epithelial cells exposed to $S$. aureus clinical isolates [11]. However, a role for RANTES in the immune response to SABSI has not been previously reported. RANTES' main function is as a chemotactic agent for leucocytes facilitating their recruitment to infection sites [18]. RANTES production is thought to be induced by TNF- $\alpha$ and IL- $1 \alpha$, both of which are produced in response to $S$. aureus components (e.g. peptidoglycan, LTA) [19,20]. Our observation of a significant rise in RANTES levels in all patient groups by day seven, suggests that RANTES production is stimulated by $S$. aureus in vivo and it may have a functional role in the immune response to this pathogen. It appears that once produced, circulating RANTES levels remain high. Furthermore, mean levels remained elevated in those with complicated SABSI up to 14 days following diagnosis. The purpose of this persistence is not clear but it may reflect the ongoing response to persistent SABSI.

Leptin levels were significantly higher in uncomplicated versus complicated SABSI on the day of laboratory diagnosis $(p \leq 0.05)$ and a similar trend was identified on day seven following diagnosis $(p=0.071)$, indicating that patients with more severe infection had a sustained attenuated leptin response. Leptin is reported to have both beneficial and detrimental effects for the host in the setting of sepsis. It stimulates proliferation of lymphohaematopoetic cells and increases the phagocytic activity of macrophages which may enhance host eradication of $S$. aureus but it can also activate endothelial cells resulting in a severe sepsis phenotype $[21,22]$. Both negative and 
positive correlations between leptin levels and clinical outcome in patients with sepsis have been reported [22,23]. The present study supports the suggestion that higher leptin levels are protective. Decreased leptin levels in patients with complicated infection may contribute to poor clinical outcome by reducing the host's ability to eradicate the infection. These findings support the possibility that administration of leptin may benefit patients with severe SABSI as one animal study reported that exogenous administration of leptin reduced the severity of $S$. aureus-induced septic arthritis in two mouse models [24].

We did not find evidence that GRO- $\gamma$ levels were altered in patients with a complicated course of infection. However, our data suggest that this cytokine may play a role in the response to MRSA infection specifically. The cause and clinical implications of this finding are unclear. Bacterial components, such as peptidoglycan and LTA that stimulate GRO- $\gamma$ production via IL-1 and TNF- $\alpha$ [20] are common to MRSA and MSSA. Furthermore, the chemoattractant properties of GRO- $\gamma$ are equally important in MRSA and MSSA (BSI). It has been shown that mecA augments the expression of virulence genes such as fnb [25] and increased expression of $\alpha$-toxin and phenol soluble modulin has been shown in community-acquired MRSA [26]. It is therefore possible that differential host immune responses such as increased $\mathrm{GRO}-\gamma$ production may result indirectly from increased expression of virulence genes in MRSA.

There were limitations to this study. Despite extension to two centres, only 61 patients were recruited over 28 months, providing a relatively small study size. There were logistical issues related to obtaining samples from very ill patients or the movement or discharge of patients before samples could be taken. Samples at 14 days after diagnosis were only taken from inpatients with complicated SABSI and not those discharged by this time. Moreover, a true baseline cytokine level was not obtained as this would require screening large numbers of patients before the development and laboratory diagnosis of SABSI. Complicated SABSI was under-represented because of the difficulty obtaining patient consent or assent from relatives of very ill or rapidly deteriorating patients. The contribution of comorbid conditions associated with SABSI (e.g. concurrent infection, autoimmune conditions, recent surgery etc.) which was not part of this study, to the cytokine response cannot be excluded. Finally, the first blood sample was taken on the day of laboratory diagnosis of SABSI and not when SABSI was first suspected on clinical grounds. This time difference was variable but may have been at least two days, over which cytokine levels may have changed.

\section{Conclusions}

In conclusion, differential expression of certain cytokines, such as IL-6 and leptin occurs in patients with different clinical courses of SABSI. While acknowledging that further studies on larger patient groups are required, these cytokines may be considered as potential prognostic markers or potentially be modified to minimise the adverse effects of $S$. aureus infection in vulnerable patients. Additionally, the increased expression of RANTES in all groups over the course of SABSI suggests that it may play a novel role in response to this pathogen.

\section{Abbreviations}

SABSI: Staphylococcus aureus bloodstream infection; RANTES: Regulated upon activation, normal T-cell expressed; GRO: Growth regulated oncogene; IE: Infective endocarditis; MRSA: Methicillin-resistant S. aureus; MSSA: Methicillin-susceptible S. aureus; TNF: Tumour necrosis factor; IL: Interleukin; IFN: Interferon; LTA: Lipoteichoic acid; ELISA: Enzyme linked immunosorbant assay; BH: Beaumont Hospital; MMUH: Mater Misericordiae University Hospital.

\section{Competing interests}

$\mathrm{HH}$ has received research support from Steris Corporation, Inov8 Science, Pfizer \& Cepheid in recent years. He has also recently received lecture \& other fees from Novartis, Astellas and AstraZeneca. No conflict of interest is reported by the other co-authors.

\section{Authors' contributions}

SMN participated in the conduct of the experiments described and produced the first draft. AT and JO'G participated in patient recruitment and sample collection, MH and ML facilitated the study, guided the ethics application at MMUH and provided critical appraisal of the manuscript, CG participated in data analysis and interpretation of the data and provided critical appraisal of the manuscript. $\mathrm{HH}$ and DFH conceived of the study, DH directed the experimental work and redrafted the manuscript. All authors approved the final manuscript.

\section{Acknowledgments}

This work was supported by Pfizer Ireland [Educational Award, No.WS 376235].

\section{Author details}

'Department of Clinical Microbiology, RCSI Education and Research Centre, Beaumont Hospital, Dublin 9, Ireland. 'Department of Microbiology, Beaumont Hospital, Dublin, Ireland. ${ }^{3}$ Department of Microbiology, Mater Misericordiae University Hospital, Dublin, Ireland. ${ }^{4}$ Department of Medicine, Royal College of Surgeons in Ireland, Dublin, Ireland.

Received: 26 November 2013 Accepted: 22 October 2014

Published online: 15 November 2014

\section{References}

1. Lindsay JA, Holden MT: Understanding the rise of the superbug: investigation of the evolution and genomic variation of Staphylococcus aureus. Funct Integr Genomics 2006, 6(3):186-201.

2. Fowler VG Jr, Nelson CL, Mclntyre LM, Kreiswirth BN, Monk A, Archer GL, Federspiel J, Naidich S, Remortel B, Rude T, Brown P, Reller LB, Corey GR, Gill SR: Potential associations between hematogenous complications and bacterial genotype in Staphylococcus aureus infection. J Infect Dis 2007, 196(5):738-747.

3. Takata T, Miyazaki M, Futo M, Hara S, Shiotsuka S, Kamimura H, Yoshimura H, Matsunaga A, Nishida T, Ishikura H, Tamura K, Tsuji BT: Presence of both heterogeneous vancomycin-intermediate resistance and beta-lactam antibiotic-induced vancomycin resistance phenotypes is associated with the outcome in methicillin-resistant Staphylococcus aureus bloodstream infection. Scand J Infect Dis 2013, 45(3):203-212.

4. Verkaik NJ, Dauwalder O, Antri K, Boubekri I, de Vogel CP, Badiou C, Bes M, Vandenesch F, Tazir M, Hooijkaas H, Verbrugh HA, van Belkum A, Etienne J, Lina G, Ramdani-Bouguessa N, van Wamel WJ: Immunogenicity of toxins during Staphylococcus aureus infection. Clin Infect Dis 2010, 50(1):61-68.

5. Santos SS, Brunialti MK, Rigato O, Machado FR, Silva E, Salomao R: Generation of nitric oxide and reactive oxygen species by neutrophils 
and monocytes from septic patients and association with outcomes. Shock 2012, 38(1):18-23.

6. Fowler VJ, Justice A, Moore C, Benjamin DK Jr, Woods CW, Campbell S, Reller LB, Corey GR, Day NP, Peacock SJ: Risk factors for hematogenous complications of intravascular catheter-associated Staphylococcus aureus bacteremia. Clin Infect Dis 2005, 40(5):695-703.

7. Wang JE, Jorgensen PF, Almlof M, Thiemermann C, Foster SJ, Aasen AO, Solberg R: Peptidoglycan and lipoteichoic acid from Staphylococcus aureus induce tumor necrosis factor alpha, interleukin 6 (IL-6), and IL-10 production in both $\mathrm{T}$ cells and monocytes in a human whole blood model. Infect Immun 2000, 68(7):3965-3970.

8. Friedman ND, Kaye KS, Stout JE, McGarry SA, Trivette SL, Briggs JP, Lamm W, Clark C, MacFarquhar J, Walton AL, Reller LB, Sexton DJ: Health care-associated bloodstream infections in adults: a reason to change the accepted definition of community-acquired infections. Ann Intern Med 2002, 137(10):791-797.

9. Peres AG, Madrenas J: The broad landscape of immune interactions with Staphylococcus aureus: from commensalism to lethal infections. Burns 2013, 39(3):380-388.

10. Yao L, Bengualid V, Lowy FD, Gibbons JJ, Hatcher VB, Berman JW: Internalization of Staphylococcus aureus by endothelial cells induces cytokine gene expression. Infect Immun 1995, 63(5):1835-1839.

11. Strindhall J, Lindgren PE, Lofgren S, Kihlstrom E: Clinical isolates of Staphylococcus aureus vary in ability to stimulate cytokine expression in human endothelial cells. Scand J Immunol 2005, 61(1):57-62.

12. Berbari E, Mabry T, Tsaras G, Spangehl M, Erwin PJ, Murad MH, Steckelberg J, Osmon D: Inflammatory blood laboratory levels as markers of prosthetic joint infection: a systematic review and meta-analysis. J Bone Joint Surg 2010, 92(11):2102-2109.

13. Damas $P$, Ledoux D, Nys M, Vrindts $Y$, De Groote D, Franchimont P, Lamy M: Cytokine serum level during severe sepsis in human IL- 6 as a marker of severity. Ann Surg 1992, 215(4):356-362

14. Hack CE, De Groot ER, Felt-Bersma RJ, Nuijens JH, Strack Van Schijndel RJ, Eerenberg-Belmer AJ, Thijs LG, Aarden LA: Increased plasma levels of interleukin-6 in sepsis. Blood 1989, 74(5):1704-1710.

15. Uusitalo-Seppala R, Koskinen $P$, Leino A, Peuravuori H, Vahlberg T, Rintala EM: Early detection of severe sepsis in the emergency room: diagnostic value of plasma C-reactive protein, procalcitonin, and interleukin-6. Scand J Infect Dis 2011, 43(11-12):883-890.

16. Feezor RJ, Oberholzer C, Baker HV, Novick D, Rubinstein M, Moldawer LL, Pribble J, Souza S, Dinarello CA, Ertel W, Oberholzer A: Molecular characterization of the acute inflammatory response to infections with gram-negative versus gram-positive bacteria. Infect Immun 2003, 71(10):5803-5813.

17. Jekarl DW, Lee SY, Lee J, Park YJ, Kim Y, Park JH, Wee JH, Choi SP. Procalcitonin as a diagnostic marker and IL-6 as a prognostic marker for sepsis. Diagn Microbiol Infect Dis 2013, 75(4):342-347.

18. Venge J, Lampinen M, Hakansson L, Rak S, Venge P: Identification of IL-5 and RANTES as the major eosinophil chemoattractants in the asthmatic lung. J Allergy Clin Immunol 1996, 97(5):1110-1115.

19. Megyeri K, Mandi Y, Degre M, Rosztoczy I: Induction of cytokine production by different Staphylococcal strains. Cytokine 2002, 19(4):206-212

20. Kanangat S, Postlethwaite A, Cholera S, Williams L, Schaberg D: Modulation of virulence gene expression in Staphylococcus aureus by interleukin-1 beta: novel implications in bacterial pathogenesis. Microbes Infect 2007, 9(3):408-415.

21. Arnalich F, Lopez J, Codoceo R, Jimnez M, Madero R, Montiel C: Relationship of plasma leptin to plasma cytokines and human survivalin sepsis and septic shock. J Infect Dis 1999, 180(3):908-911.

22. Bornstein SR, Licinio J, Tauchnitz R, Engelmann L, Negrao AB, Gold P, Chrousos GP: Plasma leptin levels are increased in survivors of acute sepsis: associated loss of diurnal rhythm, in cortisol and leptin secretion. J Clin Endocrinol Metab 1998, 83(1):280-283.

23. Shapiro NI, Khankin EV, Van Meurs M, Shih SC, Lu S, Yano M, Castro PR, Maratos-Flier E, Parikh SM, Karumanchi SA, Yano K: Leptin exacerbates sepsis-mediated morbidity and mortality. J Immunol 2010, 185(1):517-524.

24. Hultgren $\mathrm{OH}$, Tarkowski A: Leptin in septic arthritis: decreased levels during infection and amelioration of disease activity upon its administration. Arthritis Res 2001, 3(6):389-394.
25. Vaudaux PE, Monzillo V, Francois P, Lew DP, Foster TJ, Berger-Bachi B: Introduction of the mec element (methicillin resistance) into Staphylococcus aureus alters in vitro functional activities of fibrinogen and fibronectin adhesins. Antimicrob Agents Chemother 1998, 42(3):564-570.

26. Otto M: Basis of virulence in community-associated methicillin-resistant Staphylococcus aureus. Annu Rev Microbiol 2010, 64:143-162.

doi:10.1186/s12879-014-0580-6

Cite this article as: McNicholas et al:: Cytokine responses to Staphylococcus aureus bloodstream infection differ between patient cohorts that have different clinical courses of infection. BMC Infectious Diseases 2014 14:580.

\section{Submit your next manuscript to BioMed Central and take full advantage of:}

- Convenient online submission

- Thorough peer review

- No space constraints or color figure charges

- Immediate publication on acceptance

- Inclusion in PubMed, CAS, Scopus and Google Scholar

- Research which is freely available for redistribution 